\title{
Prenatal Dexamethasone Exposure Increases the Susceptibility to Autoimmunity in Offspring Rats by Epigenetic Programing of Glucocorticoid Receptor
}

\author{
Yanhong Sun, ${ }^{1,2}$ Xiaoyan Wan, ${ }^{3,4}$ Juan Ouyang, ${ }^{1}$ Renfeng Xie, \\ Xueping Wang, ${ }^{5}$ and Peisong Chen ${ }^{1}$ \\ ${ }^{1}$ Department of Laboratory Medicine, The First Affiliated Hospital of Sun Yat-sen University, Zhongshan Road 2, \\ Guangzhou 510080, China \\ ${ }^{2}$ Department of Laboratory Medicine, Huangpu Division, The First Affiliated Hospital of Sun Yat-sen University, \\ Huangpu East Road 3762, Guangdong, Guangzhou 510700, China \\ ${ }^{3}$ Family Planning Research Institute, Tongji Medical College, Huazhong University of Science and Technology, Hubei, \\ Wuhan 430000, China \\ ${ }^{4}$ Center of Reproductive Medicine, Tongji Medical College, Huazhong University of Science and Technology, Hubei, \\ Wuhan 430000, China \\ ${ }^{5}$ Department of Laboratory Medicine, Sun Yat-sen University Cancer Center, Dongfeng East Road 651, Guangzhou 510060, China
}

Correspondence should be addressed to Peisong Chen; chps@mail3.sysu.edu.cn

Received 6 September 2016; Revised 22 November 2016; Accepted 27 November 2016

Academic Editor: You-Lin Tain

Copyright (C) 2016 Yanhong Sun et al. This is an open access article distributed under the Creative Commons Attribution License, which permits unrestricted use, distribution, and reproduction in any medium, provided the original work is properly cited.

Objective. Prenatal glucocorticoids (GC) can induce long term effects on offspring health. However, reports and related studies regarding the prolonged effects of prenatal GC on the development of autoimmunity are limited. Here, we aimed to explore the immunological effects of dexamethasone (DEX) exposure on young adults and whether glucocorticoid receptor (GR) is involved in this process. Methods. Wistar rats were given DEX during pregnancy. Susceptibility to autoimmunity in offspring was assessed using experimental autoimmune encephalomyelitis (EAE) and adjuvant-induced arthritis (AIA) animal models. To reveal the possible mechanism, glucocorticoid response, GR expression, and methylation status were measured in peripheral blood mononuclear cells (PBMCs). Results. Our results showed that the DEX-treated rats had greater susceptibility to EAE (100\% versus $62.5 \%, P<0.05)$ and AIA (63.6\% versus $0 \%, P<0.05)$ than saline control group. Glucocorticoid response and GR expression were decreased in DEX rats. Significant difference was also found in the methylation levels of GR exon 1-10 to exon 1-11 region. Conclusions. Prenatal DEX administration increases the susceptibility to autoimmune diseases, which is potentially mediated by programming GR methylation status and glucocorticoid sensitivity.

\section{Introduction}

Glucocorticoids are essential for the maturation of fetal organ systems and normal development. During the course of human pregnancy, glucocorticoid (GC) treatment is often given when preterm delivery is expected. This treatment is successful in maturing the fetal lung and decreasing the occurrence of respiratory distress syndrome. However, emerging evidence indicates that antenatal glucocorticoids may elicit fetal programming that leads to adverse effects in adulthood, like hypertension, metabolic syndrome, and allergy [1-3]. This intergenerational transmission of the GC effects is mainly mediated by epigenetic mechanisms $[2,4$, 5]. In animals, the effects of prenatal GC treatment on the development of the immune system have been evaluated in small series, which indicated that prenatal GC treatment may result in the alteration of inflammatory factors [6,7], and thus contribute to the disturbance of immune system. However, reports about the mechanism linked prenatal GC exposure and autoimmunity are few. GC and glucocorticoid receptor 
(GR) signaling play a critical role in regulating the immune system. Our previous studies indicated that autoimmunity is closely related with glucocorticoid resistance and GR dysfunction, which potentially serve as the mechanism of autoimmune disease [8,9]. Taking these clues together, we hypothesize that prenatal GC exposure may contribute to the etiology of autoimmune disease through epigenetic modification of GR and glucocorticoid response [9]. The current study was designed to examine our hypothesis, which may provide insight into the early pathogenicity of certain autoimmune diseases (e.g., systemic lupus erythematosus, multiple sclerosis, and diabetes) and facilitate the development of preventative and therapeutic strategies.

\section{Materials and Methods}

2.1. Study Design. All experiments were performed in accordance with guidelines developed by the Chinese Council on Animal Care, and the protocol was approved by Sun Yatsen University Animal Care Committee. Wistar rats (200$250 \mathrm{~g})$ and guinea pigs (300-500 g) were obtained from Sun Yat-sen University (Guangzhou, China) and maintained in an animal facility at $25 \pm 3^{\circ} \mathrm{C}$, with a relative humidity of $55 \pm 3 \%$, in a $12 \mathrm{~h}$ light $/ 12 \mathrm{~h}$ dark cycle (the light is turned on at 8 am and turned off at $8 \mathrm{pm}$ ), with ad libitum access to food and tap water. Male Wistar rats were caged with individual females until mating was confirmed. After mating, female rats were randomly divided into two groups ( $n=8$ /group): DEX-treated and saline-treated. Rats were subcutaneously administered dexamethasone sodium phosphate $0.1 \mathrm{mg} / \mathrm{kg} / \mathrm{d}$ (Tianxin Pharmaceutical Factory, China, with a primary concentration of $5 \mathrm{mg} / \mathrm{mL}$, diluted into $2 \mathrm{mg} / \mathrm{mL}$ with sterile saline when using, the concentration makes the injection volume half of rats weight) or saline $0.05 \mathrm{~mL} / \mathrm{kg} / \mathrm{d}$ post aurem, from gestational days 14 to 20 to conduct a prenatal DEX exposure model or vehicle control. The injection volume varied with $0.15 \sim 0.19 \mathrm{~mL}$ according to the weight of rats.

Pups were sexed at birth, counted, and weighed. Each litter was culled to six pups ( 3 male and 3 female), and all pups were kept with their birth mother until weaning on postnatal day (PD) 25. On PD50, offspring rats were randomly chosen for different experimental procedures. Health of the animals was monitored every day.

Researchers were all adequately trained and experienced in the recognition of the signs demonstrated in the experiment. Before any punctures or operations, all rats and guinea pigs (preparing for extracting antigen to induce EAE) were anesthetized with $7 \%$ chloral hydrate administrated intraperitoneally $(0.5 \mathrm{~mL} / 100 \mathrm{~g})$, and experiment started only when animals were deeply anesthetized. After operation, animals were placed in soft and clean cages and watched carefully four times a day. If rats were found unable to access food after operation or unable to recover to stand up following experiments, they got human endpoints and were anesthetized with $20 \%$ chloral hydrate $(1 \mathrm{~mL} / 100 \mathrm{~g})$ administrated intraperitoneally.

2.2. Assessment of EAE Susceptibility. EAE was induced in 10 DEX rats (5 male and 5 female) and 8 saline rats (4 male and 4 female) using previously established procedure with modifications [10]. Briefly, spinal cords were dissected from guinea pigs ( $350 \pm 20 \mathrm{~g}$ ) and homogenized on ice in an equal volume $(\mathrm{w} / \mathrm{v})$ of sterile saline with an electronic homogenizer. The spinal cords were then emulsified with an equal volume of complete Freund's adjuvant containing $6 \mathrm{mg} / \mathrm{mL}$ heat-killed Butyribacteriumrettgeri (Difco, USA) at $4^{\circ} \mathrm{C}$. On PD50, EAE was induced by subcutaneous injection of the emulsion with the volume of $0.1 \mathrm{~mL}$ per pad at all four footpads. Rats were then intraperitoneally injected with levomisole $(10 \mathrm{mg} / \mathrm{kg}$; Sigma-Aldrich, USA) after 0,24 , and 48 h of immunization. After immunization, body weights and neurological signs of rats were examined daily. Clinical scores of these models were evaluated according to a standard scale [11]. On day 30 after immunization, rats were sacrificed, and their lumbar cords were dissected for pathological examination.

2.3. Assessment of AIA Susceptibility. AIA was induced in 11 DEX rats ( 6 male and 5 female) and 8 saline rats ( 4 male and 4 female) following a former established protocol with modifications [12]. Briefly, on PD50, $0.15 \mathrm{~mL}$ of complete Freund's adjuvant containing $10 \mathrm{mg} / \mathrm{mL}$ heat-killed $M$. tuberculosis H37Ra (Chondrex, USA) was subcutaneously injected into one hind paw pad of each rat. One week later, rats received a second injection of the same volume in the other hind paw pad. Following the second immunization, rats were visually inspected daily for signs of arthritis, erythema, and skin nodules, especially on ears and tails. Clinical scores that included arthritis and systemic symptoms were evaluated according to a standard scale by two observers blinded to group-identifying information [13]. Maximum scores, calculated at illness peak by adding the largest scores for each metric, were compared between the two groups. Twenty days after the second immunization, rats were sacrificed and their joints were removed for pathological examination. All histological procedures were performed by the histology laboratory of The First Affiliated Hospital of Sun Yat-sen University, China.

2.4. Assay of Peripheral Blood Mononuclear Cell Sensitivity to GC. The sensitivity of peripheral blood mononuclear cells (PBMCs) to GC was assessed by measuring their apoptotic response to stimulation with DEX for $18 \mathrm{~h}$. The blood from 15 DEX rats ( 7 male and 8 female) and 12 saline rats (6 male and 6 female) on PD50 was collected by cardiac puncture into heparinized vacutioners (BD Biosciences, USA). Cardiac puncture was performed under the anesthesia of intraperitoneal injection of $7 \%$ chloral hydrate $(0.5 \mathrm{~mL} / 100 \mathrm{~g})$. Rats died due to cardiac puncture but not euthanasia. We have described the possibility of animal death without euthanasia in our study protocol submitted to our institutional committee. PBMCs were immediately purified with Ficoll (KeyGen, China) and incubated with $10 \mathrm{mmol} / \mathrm{L}$ dexamethasone (Sigma-Aldrich, USA, dexamethasone powder dissolved with absolute alcohol) at $37^{\circ} \mathrm{C}$ in a humidified atmosphere containing $5 \% \mathrm{CO}_{2}$. After $18 \mathrm{~h}$, cells were harvested, stained with Annexin-V to detect cell membrane phosphatidylserine exposure (a measure of apoptosis), and costained with propidium iodide (PI; Keygen, China) to detect necrosis. 
TABLe 1: Primers for real-time PCR.

\begin{tabular}{|c|c|c|c|c|}
\hline Name & Accession number & Primers & Length (bp) & $T_{m}\left({ }^{\circ} \mathrm{C}\right)$ \\
\hline \multirow{2}{*}{ GAPDH } & \multirow{2}{*}{ NM_017008 } & F: $5^{\prime}$-GCAAGTTCAACGGCACAG-3' & \multirow{2}{*}{140} & \multirow{2}{*}{60.5} \\
\hline & & R: $5^{\prime}$-GCCAGTAGACTCCACGACAT-3' & & \\
\hline \multirow{2}{*}{ GR } & \multirow{2}{*}{ AJ271870 } & F: $5^{\prime}$-ACTGCTGGAGGTGATTGA-3' & \multirow{2}{*}{157} & \multirow{2}{*}{53.2} \\
\hline & & R: 5'-GTTTCTGAAGCCTGGTAT-3' & & \\
\hline \multirow{2}{*}{ Exon 1-1 } & \multirow{2}{*}{ AJ271870 } & F: 5'-CCAGGAAGAAGTTTCAGAT-3' & \multirow{2}{*}{195} & \multirow{2}{*}{57} \\
\hline & & R: 5'-GATGCAGAAACCTTGACTGT-3' & & \\
\hline \multirow{2}{*}{ Exon 1-4 } & \multirow{2}{*}{ AJ271870 } & F: $5^{\prime}$-CCCCAAAGCAACACCGTA- $3^{\prime}$ & \multirow{2}{*}{186} & \multirow{2}{*}{56.1} \\
\hline & & R: 5'-CAGGGACTTCGTCTCTACCAG-3' & & \\
\hline \multirow{2}{*}{ Exon 1-5 } & \multirow{2}{*}{ AJ271870 } & F: $5^{\prime}$-GGCATGCAACTTCCTCCGAGTTTA-3' & \multirow{2}{*}{176} & \multirow{2}{*}{55.4} \\
\hline & & R: 5'-AGGGCGAAGATGCAGAAACCTTGA-3' & & \\
\hline \multirow{2}{*}{ Exon 1-6 } & \multirow{2}{*}{ AJ271870 } & F: 5'-GGGCTCACATTAATATTTGC-3' & \multirow{2}{*}{149} & \multirow{2}{*}{54.3} \\
\hline & & R: 5'-AAACCTTGACTGTAGCTCCTC-3' & & \\
\hline \multirow{2}{*}{ Exon 1-7 } & \multirow{2}{*}{ AJ271870 } & F: $5^{\prime}$-AAGAAACTCGGTTTCCCT-3' & \multirow{2}{*}{167} & \multirow{2}{*}{55} \\
\hline & & R: 5' -AACCTTGACTGTAGCTCCTC-3' & & \\
\hline \multirow{2}{*}{ Exon 1-8 } & \multirow{2}{*}{ AJ271870 } & F: $5^{\prime}$-CGACAGTCGCCAACAGTTAAT-3' & \multirow{2}{*}{81} & \multirow{2}{*}{57.3} \\
\hline & & R: 5'-TGCCAGGGACTTCGTCTCTA-3' & & \\
\hline \multirow{2}{*}{ Exon 1-9 } & \multirow{2}{*}{ AJ271870 } & F: 5'-CGGGGACGGATTCTAAT-3' & \multirow{2}{*}{164} & 551 \\
\hline & & R: 5'-AGATGCAGAAACCTTGACTGT-3' & & \\
\hline Fxon 1-10 & AI271870 & F: $5^{\prime}$-CGCCGACTTGTTTATCTG-3' & 200 & 537 \\
\hline Exon $1-10$ & A) $2 / 18 / 0$ & R: 5'-AACCTTGACTGTAGCTCCTC-3' & 200 & 33.1 \\
\hline Fxon 1-11 & AJ271870 & F: $5^{\prime}$-GCCGCAGAGAACTCAACAGT-3' & 152 & 58 \\
\hline Exon $1-11$ & A) $2 / 18 / 0$ & R: 5'-GACTGTAGCTCCTCCCCTCA-3' & 152 & 58 \\
\hline
\end{tabular}

Both Annexin-V and PI staining were measured using a flow cytometer equipped with CXP software (BD Biosciences, USA). The sensitivity of PBMCs to DEX was calculated by subtracting apoptotic PBMCs in the absence of stimulation from that observed after DEX stimulation.

2.5. GR and Methylation-Associated Genes Expression Analysis. On PD50, nine DEX rats and seven saline rats were sacrificed for molecular analysis. Total RNA was isolated from PBMCs using TRIzol (Takara, Japan) according to the manufacturer's instructions. The purity and concentration of RNA were determined using an ultraviolet spectrophotometer (NanoDrop, USA). cDNA was synthesized from total RNA using a PrimeScript RT reagent kit (Takara, Japan) and amplified by polymerase chain reaction (PCR) in duplicate (total reaction volume, $25 \mu \mathrm{L}$ ) using SYBR Premix Ex Taq (Takara, Japan) and an Applied Biosystems 7500 PCR-Cycler. The cycle parameters were $95^{\circ} \mathrm{C}$ for $30 \mathrm{~s}$ followed by 40 cycles of $95^{\circ} \mathrm{C}$ for $5 \mathrm{~s}, 59^{\circ} \mathrm{C}$ for $30 \mathrm{~s}$, and $72^{\circ} \mathrm{C}$ for $34 \mathrm{~s}$, with a final one cycle of $95^{\circ} \mathrm{C}$ for $15 \mathrm{~s}, 60^{\circ} \mathrm{C}$ for $1 \mathrm{~min}$, and $95^{\circ} \mathrm{C}$ for $15 \mathrm{~s}$. The correct size of each PCR product was verified based on a single peak in melting curves. The relative number of target genes mRNA copies was calculated with the $2^{-\Delta \Delta C t}$ method using SDS software 2.0 (Applied Biosystems, USA). The housekeeping gene glyceraldehyde-3-phosphate dehydrogenase (GAPDH) was used as an internal control. Primers for GR, GAPDH, and methylation-associated genes designed by Primer 5 software were shown in Table 1.

GR protein expression was measured by Western-blot as described before $[8,14]$. An equal amount of protein
(25 $\mu \mathrm{g} /$ lane) from each cell lysate was separated on gel electrophoresis (sodium dodecyl sulfate-polyacrylamide electrophoresis) and electrophoretically transferred to polyvinylidenedifluoride membranes (Immobilon, Millipore, Billerica, MA, USA). The membranes were blocked overnight, followed by incubation for 1-2 $\mathrm{h}$ with antibody specifically recognizing GR $(1 \mu \mathrm{g} / \mathrm{mL}$; Cat number: sc-1004; Santa Cruz Biotechnology, Inc.) and $\beta$-actin (Sigma-Aldrich, St. Louis, MO). The GR antibody recognized N-terminus of GR- $\alpha$ clone. After washing, the blots were then incubated with peroxidasecoupled secondary anti-rabbit antibody (1:20,000; SigmaAldrich, St. Louis, MO). After being washed three times with TBS-T, antigen-antibody complexes on the membranes were detected by 3,3-diaminobenzidine (Sigma-Aldrich, St. Louis, MO, USA). Densitometry (IDV-integrated density value) measurement was used to compare the Western-blot data (Alpha Innotech, San Leandro, California).

2.6. DNA Methylation Analysis. Genomic DNA was extracted from blood using a DNA extraction kit (Omega, USA). DNA concentration and quality were measured using an ultraviolet spectrophotometer (NanoDrop, USA). DNA methylation of NR3C1 within exons 1-4,1-5, 1-6, 1-7, 1-10, and 1-11, introns between exon1s, and the intron preceding exon 2 was detected using a Sequenom MassARRAY Methylation method (Boao Biotechnology Company, China) as described previously [15]. Primers were designed using Epidesigner (http://www.epidesigner.com). A 10-mer tag was added to the $5^{\prime}$ end of sense primers to balance the PCR reaction, and a T7 promoter sequence was added to the $5^{\prime}$ end of antisense 


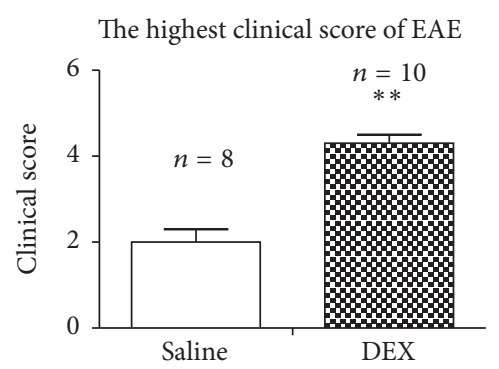

(a)

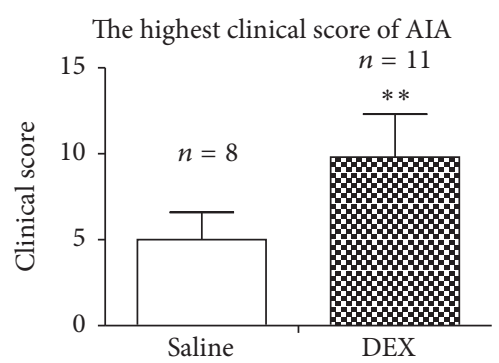

(d)

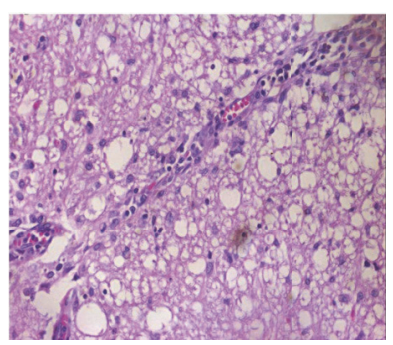

(b)

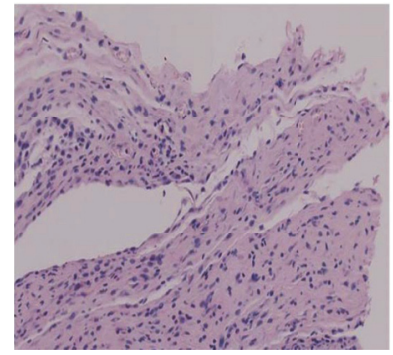

(e)

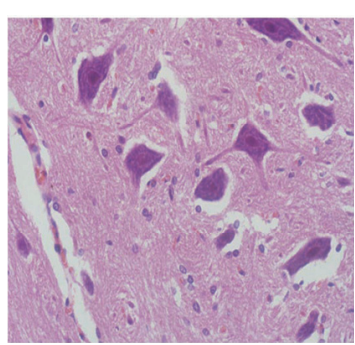

(c)

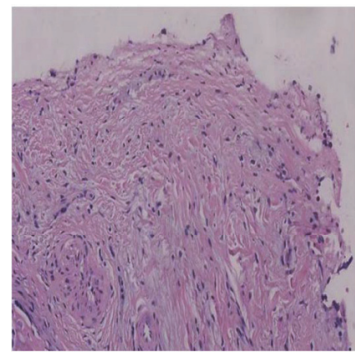

(f)

FIGURE 1: Comparative susceptibility of DEX and saline rats to EAE and AIA. (a) Comparison of clinical scores of EAE at illness peak, analyzed by Mann-Whitney nonparametric test. ${ }^{* *} P<0.01$. (b) Histopathological analysis of DEX rats. Hematoxylin and eosin staining showing an enlargement of the lumbar spinal cord in ill (DEX) rats; nervous tissue with vacuole-like structures containing many infiltrating inflammatory cells encasing vessels. (c) Histopathological analysis of saline rats. The lumbar enlargement of spinal cord from normal (saline) rats (hematoxylin and eosin staining). (d) Comparison of clinical scores of fore limb arthritis between DEX and saline rats at illness peak, analyzed by Mann-Whitney nonparametric test. ${ }^{* *} P<0.01$. (e) Histopathological analysis of DEX rats. Hematoxylin and eosin staining of an affected ankle (400x), showing many infiltrating inflammatory cells in the synovium, with vessel hyperplasia. (f) Histopathological analysis of a normal (saline) ankle.

primers for subsequent in vitro transcription. All primers are shown in Table 2. Because exon 1 contained a large number of CG sites (207), which created obstructions in primer design, we divided this region into the following seven parts and then tested each part separately: gene 1 , corresponding to the promoter of exon 1-4; gene 2, corresponding to exon $1-4$ and the promoter of exon 1-5; gene 3, corresponding to the promoter of both exon 1-5 and exon 1-6; gene 4, corresponding to the promoter of both exon 1-6 and exon 1-7; gene 5, corresponding to part of exon 1-10 and the promoter of exon 1-11; gene 6, corresponding to the promoter of both exon 1-11 and exon 2; and gene 7, corresponding to the promoter of exon 2 (Table 2).

2.7. Statistical Analysis. All calculations were performed using SPSS V.13.0 statistical software (Chicago, IL, USA). An unpaired Student's $t$-test or a Mann-Whitney nonparametric test was applied when group variances were not equal or the data was not normally distributed, to compare the means of two groups. A chi-square test was employed when analyzing proportional data. Figures were drawn using Graphpad Prism 5 (GraphPad Software Inc., San Diego, CA, USA). A P value less than 0.05 was considered significant.

\section{Results}

3.1. Characteristics at Birth. The litter size and sex ratio did not differ between DEX group and vehicle control group (litter size $11.5 \pm 0.9$ versus $10.7 \pm 1.2$, female/male ratio $1.2 \pm 0.1$ versus $1.1 \pm 0.2$ ). However, compared with saline rats, DEX rats at birth were smaller in weight $(6.2 \pm 0.8$ versus $5.31 \pm 0.6 \mathrm{~g}, P<0.01)$.

3.2. Susceptibility to EAE and AIA. Compared with saline rats, DEX rats with EAE showed higher morbidity (100\% versus $62.5 \%, P<0.05)$, earlier symptom onset $(12 \mathrm{~d}$ versus $13.5 \mathrm{~d}, P<0.05$ ), higher clinical score at illness peak ( 4.75 versus 1.93, $P<0.01$, Figure 1(a)), and more serious pathological results (Figures $1(\mathrm{~b})$ and $1(\mathrm{c})$ ). Thus, DEX rats were more susceptible to EAE than saline rats.

Compared with saline rats, DEX rats with AIA exhibited greater morbidity, measured as forelimb arthritis and systemic inflammation, than saline rats $(63.6 \%$ versus $0 \%$, $P<0.05)$. The clinical score and pathologic manifestations of DEX rats at illness peak were also higher than saline rats (9.72 versus 7.0, $P<0.01$, Figures $1(\mathrm{~d}), 1(\mathrm{e})$, and $1(\mathrm{f})$ ). In addition, early after antigen administration, focal inflammatory responses of DEX rats were more serious than those of saline rats. Onset time and hind limb arthritic morbidity were not significantly different between the two groups. Collectively, these observations indicate that DEX rats were more susceptible to AIA than saline rats.

3.3. Sensitivity of PBMCs to GC. Stimulation of PBMCs from DEX rats and saline rats with the same concentration of dexamethasone for the same period induced less apoptosis 


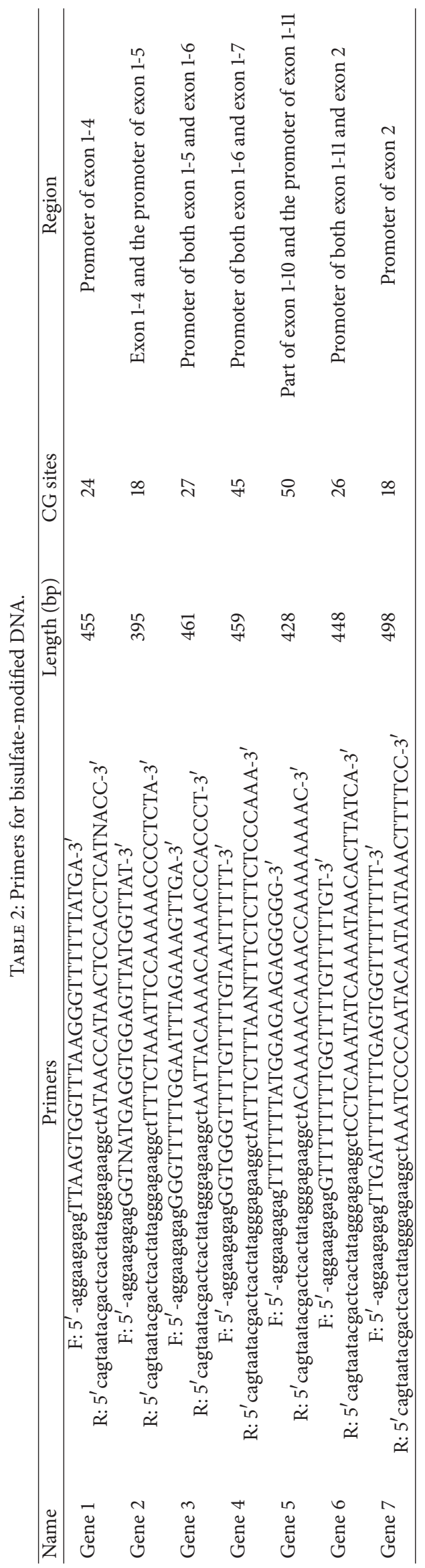


from DEX rats (mean apoptosis rate 13.30\%) than those from saline rats (mean apoptosis rate $19.15 \%, P<0.01$ ). Thus, the sensitivity of PBMCs from DEX rats to GC was lower than that from saline rats.

3.4. GR Expression in PBMCs. Since that GR gene contains 11 untranslated alternative first exons and each exon was processed by a distinct promoter, total GR mRNA and each exon 1 expression were evaluated in our study. Our data showed that total GR, exon 1-1, exon 1-6, exon 1-10, and exon 1-11 in PBMCs were lower in DEX rats than those in saline rats $(\mathrm{P}<0.05)$ (Figure 2(a)). These results were further confirmed by Western-blot, as GR protein deceased in DEX rats (Figures 2(b) and 2(c)).

3.5. DNA Methylation Analysis. The methylation level of the 207 CG sites detected in 7 regions of GR exon 1 ranged from 0 to 0.83 , with most being less than 0.3 . At most sites, methylation levels did not significantly differ between DEX and saline rats. However, in the region of exon 1-10 and exon 111 promoter, DEX rats showed higher methylation levels than saline rats (Figure $2(\mathrm{~d})$ ).

\section{Discussion}

Adverse early life events and their long term effects have attracted more and more attention in recent years. And the development of epigenetic theory and technology advance our understanding in this filed $[16,17]$. Maternal care, stress, and exogenous glucocorticoid administration are suggested to have a profound impact on offspring health $[18,19]$. However, there are few reports about the prenatal DEX exposure and its long term programming effects of autoimmunity. Here we evaluated the immune programming influenced by prenatal DEX treatment and the role of GR in this process.

To test our hypothesis, pregnant Wistar rats were subcutaneously given DEX. Wistar rats are widely used for toxicology, teratology, experimental oncology, experimental gerontology, and cardiovascular research and immunology studies $[6,20]$. Rats have a brief and accelerated childhood compared with humans and become sexually mature at about 6 weeks of age [5]. When considering the different phases of a rat's life, we chose day 50 of the rat to represent the adult stages. Two experimental autoimmune disease models, EAE and AIA, were performed with offspring rats in adulthood. Since sex hormone has some effect on the development of autoimmune disease, to avoid possible confounder from sex difference, we tried to make female/male ratio equal between groups [21]. The histopathological and clinical scores analysis between groups in EAE and AIA models also provided evidence that DEX administration during pregnancy increased susceptibility to autoimmune diseases in offspring, as DEX group showed more severe histopathological manifestation and clinical signs. During pregnancy, GC is often administrated when preterm delivery is expected, which is successful in stimulating the development of the fetal lung. However, emerging evidence indicated that prenatal GC exposure would result in prolonged adverse effects in offspring. In our animal model, the total dose of dexamethasone used in rats is much higher than that used for preterm labor owing to the continuous administration of DEX up to 7 days. Prenatal GC exposure not only existed in the treatment of preterm delivery, but also existed in mothers with various prenatal stresses $[17,22]$. In order to cover different kinds of prenatal GC exposure, we extended the period of DEX administration. Our results also indicated that the incidence of autoimmune disease (both EAE and AIA) is increased in DEX group. These results were supportive with our initial hypothesis that prenatal GC exposure increases susceptibility to autoimmune diseases.

To further explore mechanisms causing such effects, firstly, we tested the apoptotic responses of PBMCs from DEX and saline rats to dexamethasone stimulation. PBMCs, which are mainly lymphocytes and monocytes, are the target cells of immune modulation and executive of immune response. Reduced GC response in PBMCs has been reported closely related with autoimmunity [23]. Our experiments showed that dexamethasone induced a smaller increase in apoptosis in PBMCs from DEX rats than in saline rats, which indicates that PBMCs from DEX rats were more resistant to DEX. GC physically binds to GR in immune organs and cells to induce apoptosis and thereby inhibit the immune reaction. Reduced glucocorticoid response is mainly caused by GR dysfunction and abnormal expression. So we analyzed GR expression in rat PBMCs. As measured by real-time PCR and Westernblot, total GR expression in DEX rat is significantly decreased, which is supportive for the differential cell response to GC. The publications of $\mathrm{Lu}$ and Cidlowski stated that multiple functional internal ATG translation initiation codons of GR resulted in multiple GR protein forms, and GR-B, GR-C, and GR-D can represent up to $50 \%$ of the total translated $\mathrm{Gr}$ alpha protein products $[24,25]$. However, we only found one band in our plot owing to the antibody used in our study. The M20 clone used in this study has an epitope in the Nterminal region of the GR that is not present in GR-B, GR$\mathrm{C}$, and GR-D isoforms. With this antibody, we only detected the major isoform (GR-A) but not all isoforms of GR. The inability to detect these shorter GR isoforms is a limitation of our study. Since that GR exon1 contained a large number of CG sites and differentially regulated GR expression, different fragments of exonl which are rich of CG sites were also measured by real-time PCR. Our results showed that exon 1-6, exon 1-10, and exon 1-11, in PBMCs, were lower in DEX rats than those in saline rats. These results indicate that the decreased GR expression may be caused by methylation of GR exon1. We examined DNA methylation of exon 1 in the NR3C1 gene, which includes 207 CG sites covering exons $1-4,1-5,1-6,1-7,1-10$, and 1-11 and introns between them (filled with intensive GC preventing primer design, other sites were not detected), and compared their methylation level in PBMCs between saline and DEX rats. We found that a CG sites enriched region which spread from exon 1-10 to exon 1-11 was differentially methylated in DEX and saline rats. Although the exact mechanism of how this region controls GR transcription is still unknown, it is generally considered that hypermethylation in this region results in transcriptional inhibition [26, 27]. Currently, reports about the prolonged effects of prenatal GC on the methylation level in immune 


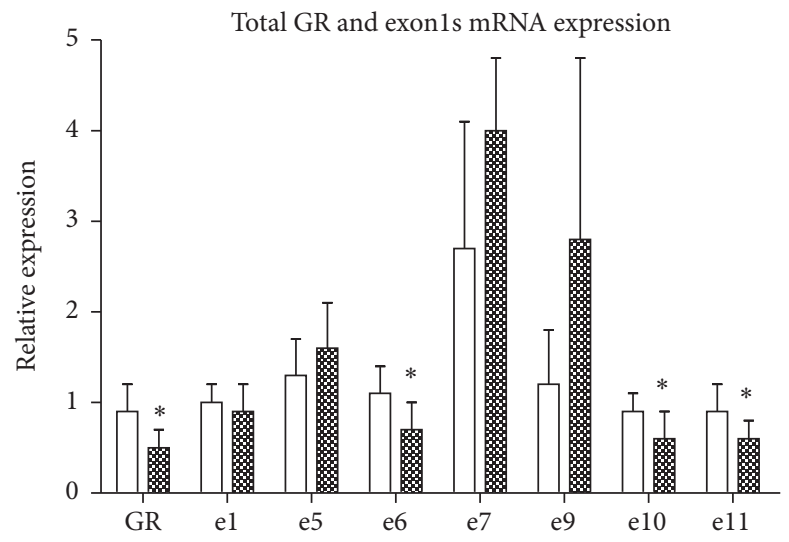

\section{Saline}

\& DEX

(a)

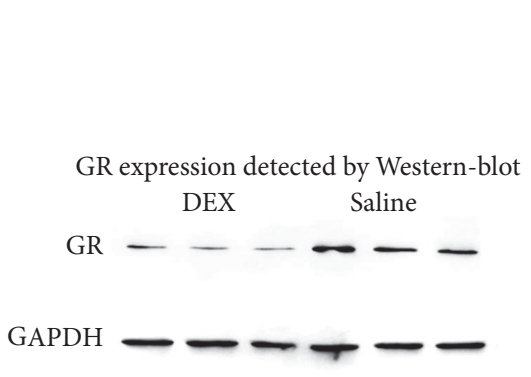

(b)

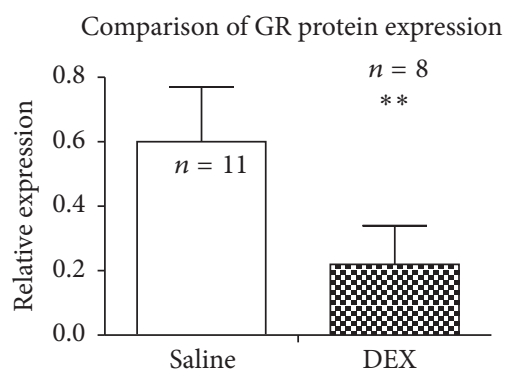

(c)

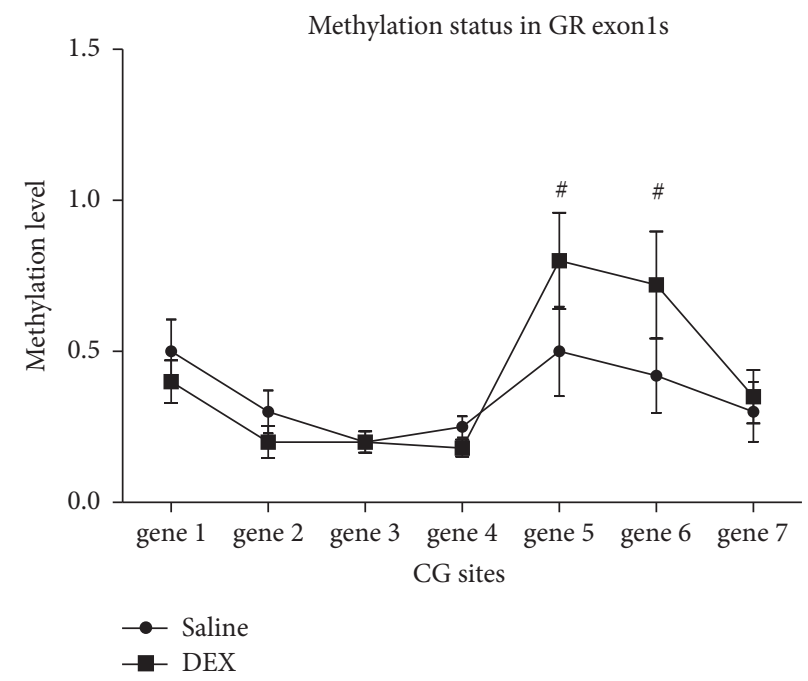

(d)

FIGURE 2: GR and different exon 1 expression and methylation. (a) mRNA expression of total GR and different exonl regions, analyzed by Mann-Whitney nonparametric test, ${ }^{*} P<0.05$. (b) GR protein expression from different groups. Lanes 1 to 3 were from DEX rats, while lanes 4 to 6 were from saline rats. (c) Relative quantitate data for GR protein expression, analyzed by Mann-Whitney nonparametric test, ${ }^{* *} P<0.01$. (d) comparison of methylation levels in exonls of GR gene between DEX and saline rats, analyzed by Mann-Whitney nonparametric test, ${ }^{\#} P<0.05$. 
cells are few. However, prenatal stress or GC exposure may alter methylation level of GR gene on hippocampus, bucca, and kidney, which are also supportive with our results [19, $28,29]$. Our data added to the current literatures that prenatal GC exposure altered the methylation level of GR and caused decreased GR expression and GC response in PBMCs, which is closely related with the development of autoimmunity.

In conclusion, we found that prenatal GC exposure increases the susceptibility to autoimmunity, which is potentially caused by epigenetically programmed GR expression and glucocorticoid response.

\section{Competing Interests}

The authors declare that they have no competing interests.

\section{Authors' Contributions}

Yanhong Sun and Xiaoyan Wan contributed equally to this work.

\section{Acknowledgments}

This study was supported by grants from NSFC (Natural Science Foundation of China) (no. 81072490 and no. 81101316).

\section{References}

[1] S. D. Bilbo and J. M. Schwarz, "Early-life programming of laterlife brain and behavior: a critical role for the immune system," Frontiers in Behavioral Neuroscience, vol. 3, article no. 14, 2009.

[2] D. R. Kim, T. L. Bale, and C. N. Epperson, "Prenatal programming of mental illness: current understanding of relationship and mechanisms," Current Psychiatry Reports, vol. 17, no. 2, article 546, 2015.

[3] A. Vaag, C. Brøns, L. Gillberg et al., "Genetic, nongenetic and epigenetic risk determinants in developmental programming of type 2 diabetes," Acta Obstetricia et Gynecologica Scandinavica, vol. 93, no. 11, pp. 1099-1108, 2014.

[4] T. L. Bale, "Sex differences in prenatal epigenetic programing of stress pathways," Stress, vol. 14, no. 4, pp. 348-356, 2011.

[5] M. Darnaudéry and S. Maccari, "Epigenetic programming of the stress response in male and female rats by prenatal restraint stress," Brain Research Reviews, vol. 57, no. 2, pp. 571-585, 2008.

[6] H.-R. Yu, H.-C. Kuo, C.-C. Chen et al., "Prenatal dexamethasone exposure in rats results in long-term epigenetic histone modifications and tumour necrosis factor- $\alpha$ production decrease," Immunology, vol. 143, no. 4, pp. 651-660, 2014.

[7] H. R. Yu, Y. L. Tain, J. M. Sheen et al., "Prenatal dexamethasone and postnatal high-fat diet decrease interferon gamma production through an age-dependent histone modification in male sprague-dawley rats," International Journal of Molecular Sciences, vol. 17, no. 10, p. 1610, 2016.

[8] J. Ouyang, P. Chen, T. Jiang, Y. Chen, and J. Li, "Nuclear HSP90 regulates the glucocorticoid responsiveness of PBMCs in patients with idiopathic nephrotic syndrome," International Immunopharmacology, vol. 14, no. 3, pp. 334-340, 2012.

[9] P. Chen, T. Jiang, J. Ouyang, Y. Cui, and Y. Chen, "Epigenetic programming of diverse glucocorticoid response and inflammatory/immune-mediated disease," Medical Hypotheses, vol. 73, no. 5, pp. 657-658, 2009.
[10] G. Q. Chen, Y. Y. Chen, X. S. Wang et al., "Chronic caffeine treatment attenuates experimental autoimmune encephalomyelitis induced by guinea pig spinal cord homogenates in Wistar rats," Brain Research, vol. 1309, pp. 116-125, 2010.

[11] Y. Matsumoto, I.-K. Park, K. Hiraki, S. Ohtani, and K. Kohyama, "Role of pathogenic T cells and autoantibodies in relapse and progression of myelin oligodendrocyte glycoprotein-induced autoimmune encephalomyelitis in LEW.1AV1 rats," Immunology, vol. 128, no. 1, part 2, pp. e250-e261, 2009.

[12] U. Snekhalatha, M. Anburajan, B. Venkatraman, and M. Menaka, "Evaluation of complete Freund's adjuvant-induced arthritis in a Wistar rat model: comparison of thermography and histopathology," Zeitschrift fur Rheumatologie, vol. 72, no. 4, pp. 375-382, 2013.

[13] L. Pei-Pei, X. Guo-Xiong, and S. Shan-Shan, "Clinical manifestations and the main evaluation method on adjuvant-induced arthritis model in rats," Chinese Journal of Immunology, vol. 28, pp. 453-457, 2012.

[14] P. Chen, T. Jiang, J. Ouyang, and Y. Cui, "Glucocorticoid receptor auto-upregulation and its relation with glucocorticoid sensitivity in idiopathic nephrotic syndrome," International Urology and Nephrology, vol. 43, no. 1, pp. 167-174, 2011.

[15] L. Wang, F. Wang, J. Guan et al., "Relation between hypomethylation of long interspersed nucleotide elements and risk of neural tube defects," American Journal of Clinical Nutrition, vol. 91, no. 5, pp. 1359-1367, 2010.

[16] V. Glover, "Prenatal stress and its effects on the fetus and the child: possible Underlying biological mechanisms," Advances in Neurobiology, vol. 10, pp. 269-283, 2015.

[17] O. Babenko, I. Kovalchuk, and G. A. S. Metz, "Stress-induced perinatal and transgenerational epigenetic programming of brain development and mental health," Neuroscience \& Biobehavioral Reviews, vol. 48, pp. 70-91, 2015.

[18] F. van Bel and C. J. Heijnen, "Perinatal programming and reprogramming by glucocorticoid therapy and perinatal stress," Seminars in Fetal and Neonatal Medicine, vol. 14, no. 3, pp. 127129, 2009.

[19] T. F. Oberlander, J. Weinberg, M. Papsdorf, R. Grunau, S. Misri, and A. M. Devlin, "Prenatal exposure to maternal depression, neonatal methylation of human glucocorticoid receptor gene (NR3C1) and infant cortisol stress responses," Epigenetics, vol. 3, no. 2, pp. 97-106, 2008.

[20] H.-C. Kuo, M. M.-H. Guo, S.-F. Liu et al., "Cross-fostering increases Th1/Th2 expression in a prenatal dexamethasone exposure rat model," PLoS ONE, vol. 9, no. 12, Article ID el15554, 2014.

[21] G. Benedek, J. Zhang, S. Bodhankar et al., "Estrogen induces multiple regulatory B cell subtypes and promotes M2 microglia and neuroprotection during experimental autoimmune encephalomyelitis," Journal of Neuroimmunology, vol. 293, pp. 45-53, 2016.

[22] N. Provençal and E. B. Binder, "The effects of early life stress on the epigenome: from the womb to adulthood and even before," Experimental Neurology, vol. 268, pp. 10-20, 2015.

[23] J. Ouyang, T. Jiang, M. Tan, Y. Cui, and X. Li, "Abnormal expression and distribution of heat shock protein 90: potential etiologic immunoendocrine mechanism of glucocorticoid resistance in idiopathic nephrotic syndrome," Clinical and Vaccine Immunology, vol. 13, no. 4, pp. 496-500, 2006.

[24] N. Z. Lu and J. A. Cidlowski, "Translational regulatory mechanisms generate $\mathrm{N}$-terminal glucocorticoid receptor isoforms 
with unique transcriptional target genes," Molecular Cell, vol. 18, no. 3, pp. 331-342, 2005.

[25] F. A. Leenen, S. Vernocchi, O. E. Hunewald et al., "Where does transcription start? $5^{\prime}$-RACE adapted to next-generation sequencing," Nucleic Acids Research, vol. 44, no. 6, pp. 26282645, 2016.

[26] L. Cao-Lei, S. Suwansirikul, P. Jutavijittum, S. B. Mériaux, J. D. Turner, and C. P. Muller, "Glucocorticoid receptor gene expression and promoter CpG modifications throughout the human brain," Journal of Psychiatric Research, vol. 47, no. 11, pp. 1597-1607, 2013.

[27] Q. Sun, Y. Jia, R. Li, X. Li, X. Yang, and R. Zhao, "Breed-specific expression of GR exon $1 \mathrm{mRNA}$ variants and profile of GR promoter $\mathrm{CpG}$ methylation in the hippocampus of newborn piglets," Animal, vol. 8, no. 11, pp. 1851-1856, 2014.

[28] E. C. Braithwaite, M. Kundakovic, P. G. Ramchandani, S. E. Murphy, and F. A. Champagne, "Maternal prenatal depressive symptoms predict infant NR3C1 $1 \mathrm{~F}$ and BDNF IV DNA methylation," Epigenetics, vol. 10, no. 5, pp. 408-417, 2015.

[29] S. Petropoulos, S. G. Matthews, and M. Szyf, "Adult glucocorticoid exposure leads to transcriptional and DNA methylation changes in nuclear steroid receptors in the hippocampus and kidney of mouse male offspring," Biology of Reproduction, vol. 90, no. 2, article no. 43, 2014. 


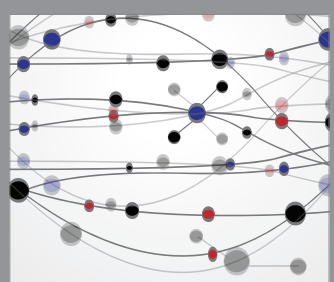

The Scientific World Journal
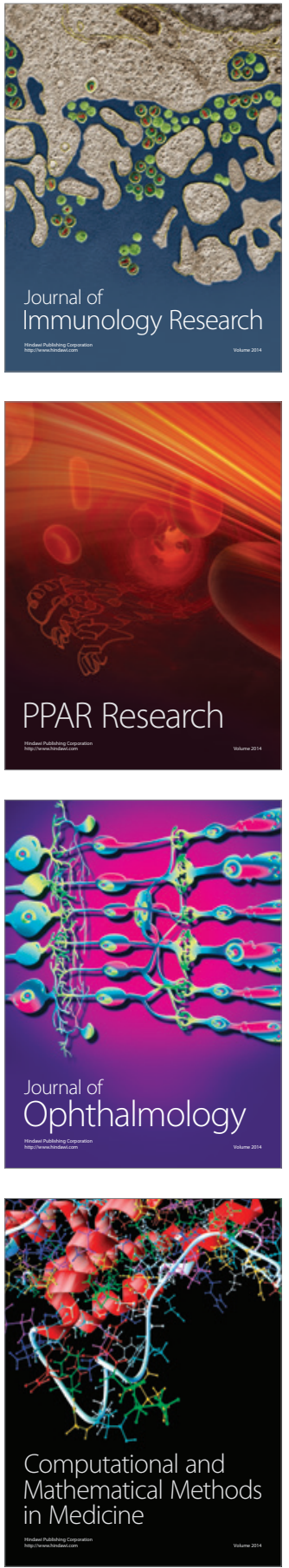

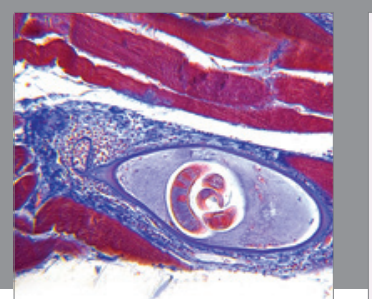

Gastroenterology Research and Practice

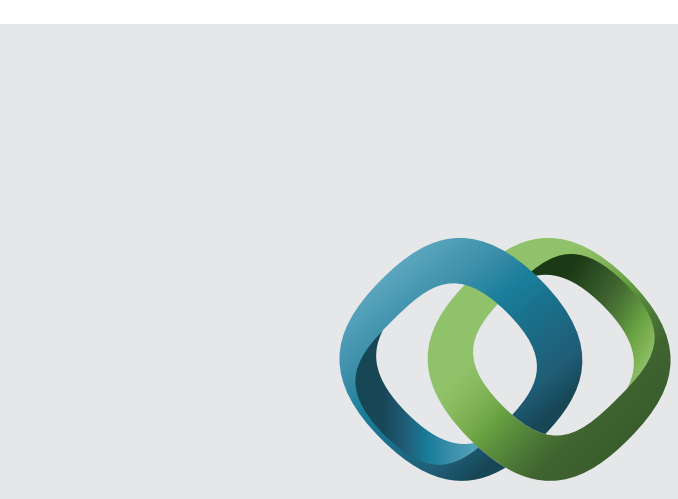

\section{Hindawi}

Submit your manuscripts at

http://www.hindawi.com
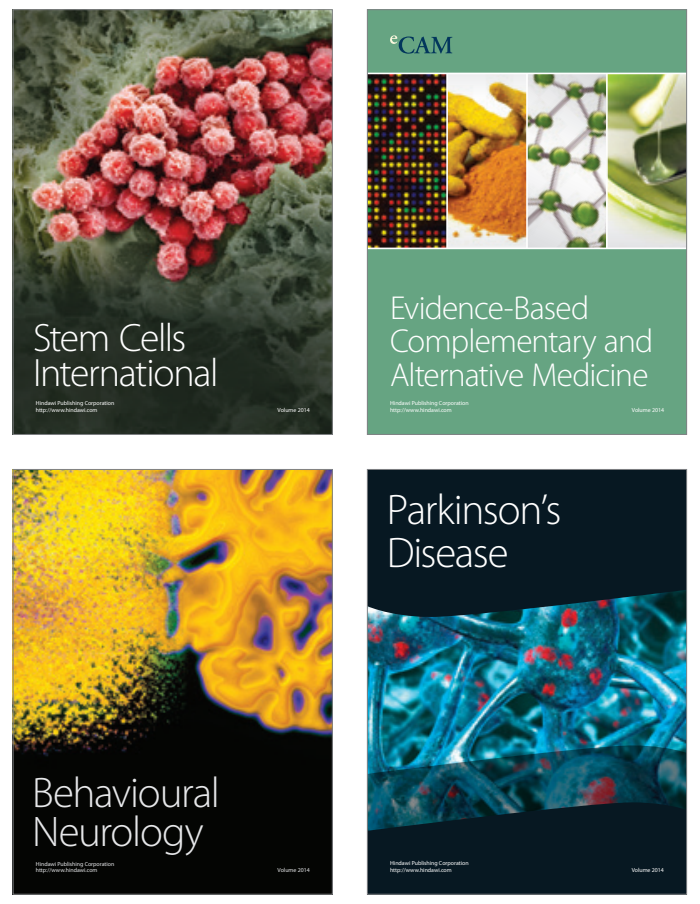
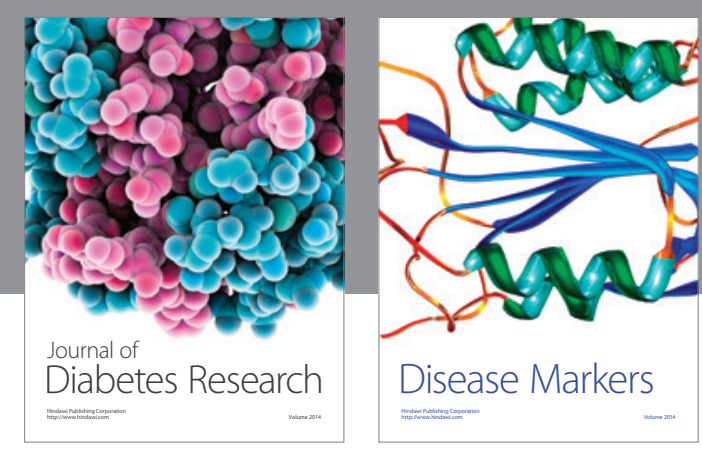

Disease Markers
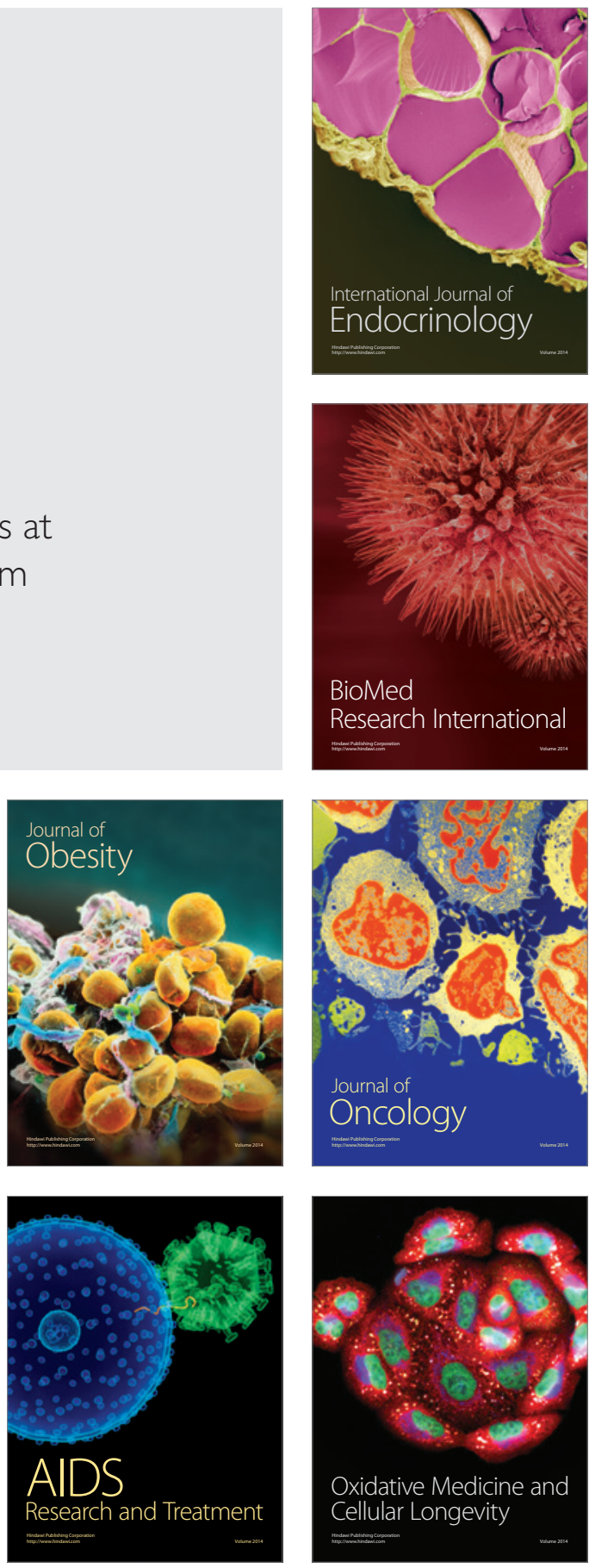